sprechend immunologisch heterogen ist diese Patientenkohorte.

In einem Mailänder MS-Zentrum wurde Natalizumab bei 84 JCV-seropositiven Patienten als PML-Prävention abgesetzt. In der Folge kam es bei $53 \%$ dieser Kohorte zu einer Re-Exazerbation der Krankheitsaktivität in den folgenden zwölf Monaten [11]. Dies liegt im Mittelfeld der in der Literatur beschriebenen Rückfallrate von $27-70 \%$ [12]. Bei sechs Patienten [7\%] trat eine Rebound-artige Zunahme der Krankheitsaktivität auf. Bei einem dieser Patienten war Natalizumab abgesetzt worden, da zuvor eine Konversion des Krankheitsverlaufes in eine sekundär-progrediente Form diagnostiziert worden war.

Am Berliner Jüdischen Krankenhaus blieben 80,3\% der Natalizumab-Patienten nach der Umsetzung auf Immunmodulatoren innerhalb der ersten sechs Monate schubfrei versus $47,3 \%$ der unbehandelten Patienten [12]. Inzwischen liegen auch erste Erfahrungen mit Patienten vor, die von Natalizumab auf Fingolimod umgestellt wurden. Hier blieben $50 \%$ nach sechs Monaten schubfrei $(\mathrm{n}=10)$.

In einer Observationsstudie am Rocky Mountain MS-Zentrum in Denver/Colorado erlitten 21/77 Patienten einen neuen

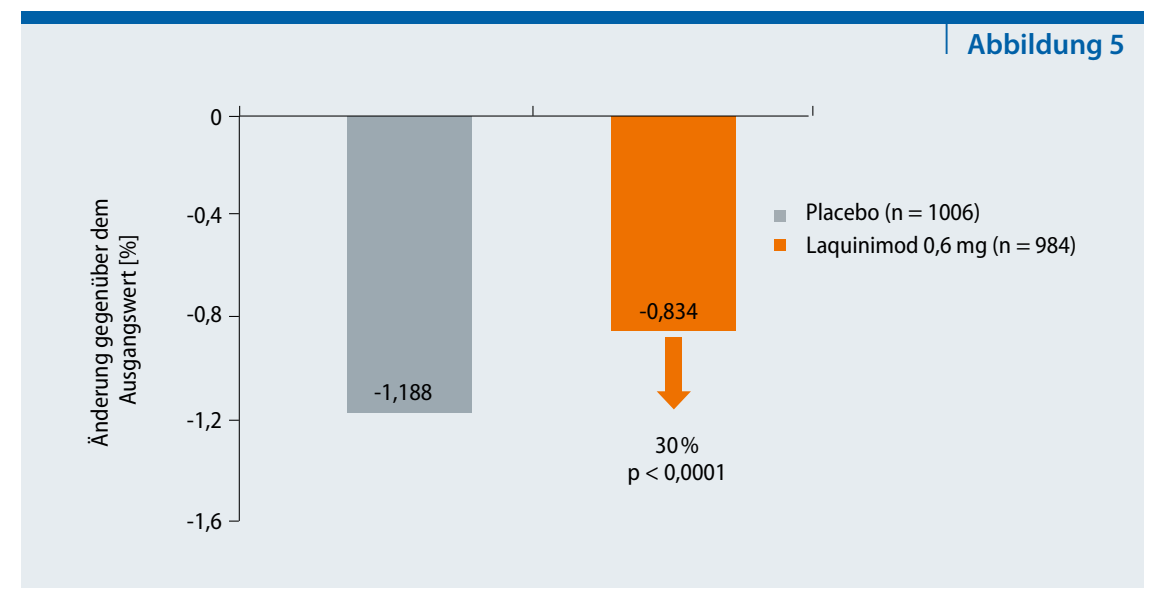

Änderungen des Gehirnvolumens (\%) unter Laquinimod und Placebo nach 24 Monaten im Vergleich zum Ausgangswert (nach [5])

klinischen Schub, wobei sich die meisten schweren Schübe bereits nach rund drei Monaten ereigneten [13]. Eine Zunahme von $\mathrm{Gd}^{+}$-Läsionen wurde nur bei Patienten mit Symptomen eines klinischen Schubes beobachtet. Potenzielle Risikofaktoren waren eine hohe Krankheitsaktivität vor Natalizumab-Therapie, lange Erkrankungsdauer (> 10 Jahre) und lange Therapiedauer mit Natalizumab. Eine Auswertung der Winthrop Universitätsklinik in Mineola/New York legt nahe, dass JCV-seropositive Patienten ein signifikant höheres Risiko eines erneuten Schubes nach Natalizumab-Absetzen besitzen [14].

Dr. Alexander Kretzschmar

Vorträge und Poster im Rahmen der 64. Jahrestagung der American Academy of Neurology (AAN), New Orleans, 21.-28. April 2012

\section{Alzheimer-Demenz \\ Neue PET-Tracer lassen auf In-vivo-Frühdiagnostik hoffen}

Mehrere Studien, die auf der AAN-Jahrestagung 2012 in New Orleans präsentiert wurden, lassen die Neurologen vom baldigen Einsatz der Bildgebung zur In-vivo-Frühdiagnostik der Alzheimer-Demenz träumen [19-21].

D e Objekte der Begierde sind die Tracersubstanzen $\left[{ }^{18} \mathrm{~F}\right]$ Flutemetamol und Florbetaben, mit deren Hilfe die Positronen-Emissions-Tomografie (PET) in die Lage versetzt werden soll, bereits im MCI-Stadium (Mild Cognitive Impairment) die Ablagerung von ß-Amyloid nachzuweisen. Studien an unterschiedlichen Patientenkollektiven - vom MCI-Stadium bis zur fortgeschrittenen Alzheimer-Demenz - zeigen eine hohe Sensitivität und Spezifität im Vergleich zu histopathologischen Befunden. versität von Pennsylvania in Philadelphia verglich bei 180 Alzheimer-Patienten im Endstadium der Erkrankung die $\left[{ }^{18} \mathrm{~F}\right]$ Flutemetamol-PET-Aufnahmen mit PostMortem-Autopsien sowie Gehirnbiopsien einer Vergleichsgruppe mit einem Normaldruck-Hydrocephalus. Die Analyse zeigte eine hohe Übereinstimmung bei der Beurteilung der Aufnahmen ( $\mathrm{k}=$ $0,86)$. Die Sensitivität und Spezifität im Vergleich mit den Biopsien betrug 100\% bzw. $96 \%$ sowie $86 \%$ bzw. $92 \%$ im Vergleich zu den Alzheimer-Autopsien.
Dr. David Wolk, Neurologe an der Uni-
Ein $\left[{ }^{18} \mathrm{~F}\right]$ Flutemetamol-PET wurde in einer weiteren Studie bei elf nicht-dementen Senioren im Alter zwischen 65 und 82 Jahren durchgeführt. Die vermehrte Tracer-Aufnahme korrelierte dabei mit kognitiven Defiziten, die auch typisch für eine Alzheimer-Demenz sind, meinte Professor Dustin Hammers, Universität von Utah, Salt Lake City.

Für den Tracer Florbetaben stellte Professor Marwan Sabbagh vom Sun Health Research Institute in Sun City die Daten einer Phase-III-Studie zum Nachweis von ß-Amyloid in der grauen Hirnsubstanz vor. Dabei wurden die PET-Scans von 204 Alzheimer-Patienten im Endstadium und zehn gesunden Kontrollen sowie PostMortem-Autopsien in sechs präselektierten „Regions-of-Interest“ (ROI) abgeglichen. Die Sensitivität betrug $77 \%$ (84\% bei Silberfärbung des Autopsiematerials), die Spezifität $94 \%(\mathrm{k}=0,66)$.

Dr. Alexander Kretzschmar 